\title{
SIGNIFICANCE OF ECCENTRIC FIXATION IN SQUINT*
}

\author{
BY
}

\author{
KENNETH WYBAR AND BARBARA THATCHER \\ From the Orthoptic Department of Moorfields Eye Hospital (High Holborn Branch)
}

\section{INTRODUCTION}

THE problem of the restoration of full vision in the squinting eye in uniocular squints is one which obtrudes itself in every phase of the treatment of strabismus. There is, of course, nothing new in the recognition of the importance of the treatment of amblyopia in squint. Occlusion of the fixing eye in cases of squint has been a recognized procedure since the middle of the 18 th century, and there is undoubted evidence that this is an effective line of treatment in the majority of such cases. On the other hand, in a certain number of cases, occlusion, even when properly applied, proves to be ineffective and it would appear that most of the unsuccessful cases are those in which there is an early age of onset of the squint and particularly when there is an eccentric type of fixation of the squinting eye. It is with cases of eccentric fixation that this paper is primarily concerned, and with a re-assessment of the widely recognized concept that in cases of amblyopia it may be possible to restore vision only to the functional level which existed at the time of onset of the amblyopia.

\section{DeVelopment of Amblyopia and Eccentric Fixation}

In order to establish a clear understanding of the development of eccentric fixation, it is important to be acquainted with the role of the retinal receptors in the visual processes. In the normal eye the retinal receptors have two distinct functions. In the first place, they have a visual acuity value, although all do not have equal functional abilities. The receptors of the macula, and more particularly those of the central zone of the macula or fovea, have a high visual acuity value and are concerned solely with central vision, whereas the receptors of the other parts of the retina have a low visual acuity value and are concerned solely with peripheral vision. It is important to note, however, that the visual function of the macula is developed gradually over the first 5 or 6 years of life. This delay in the achievement of full central vision is due partly to the fact that the macula is not completely formed at birth, but mainly to the inevitable delay in the full 
appreciation of the macular function in the visual cortex of the brain, and the basic elements of this cortical appreciation are not established until about the 6 th year of life.

In the second place, the retinal receptors have a spatial sense value, and this implies that each receptor in the retina possesses "local sign", so that excitation of each receptor is appreciated in the visual cortex in terms of projection in space, quite apart from a visual appreciation. Each retinal receptor has a unique type of spatial projection so that excitation of one receptor is discriminated from excitation of any other receptor. In the normal eye the fovea is highly endowed with local sign and its spatial sense consists of a straight-ahead type of projection, that is, a central type of fixation. The other retinal receptors have a spatial projection which is ranged round that of the fovea according to their position in the retina, so that the fovea forms the central point of reference. This is the mechanism of visual projection and it provides information on the localization of objects in space. Furthermore, it is important to realize that under normal conditions the fovea of one eye is associated with the fovea of the other eye in the visual process, so that both foveae are directed to the same object at the same time. In this way, the foveae may be regarded as corresponding retinal points and this concept of retinal correspondence must be extended to all parts of the retina so that there are points throughout the nasal part of the retina of one eye which correspond to points in the temporal part of the retina of the other eye, each pair of corresponding points lying equidistant from the foveae. Simultaneous stimulation of corresponding retinal points results in the formation of a single visual image with a single projection in space, although it should be noted that the term "retinal point" has a physiological rather than a strictly anatomical meaning, so that within a narrow area of retina (Panum's area) disparate stimulation is compatible with the formation of a single image.

The development of squint in many cases gives rise to an alteration in the function of the retinal receptors. In the first place, there may be an inhibition of the visual acuity of the retinal receptors in the macular region of the squinting eye, and this is due to a process of ocular neglect or suppression which is initiated in the cerebral cortex as an adaptation to the occurrence of confusion, a term which implies a form of diplopia arising because the macula of the squinting eye and the macula of the non-squinting eye are directed at the same time to different objects. (This differs from the usual form of diplopia in squint which is produced by stimulation of non-corresponding retinal points by the same object). This inhibition may assume two forms depending on whether the squint is uniocular or alternating in type. In a uniocular squint the inhibition is obligatory because it persists in the macula of the squinting eye irrespective of the state of activity of the macula of the non-squinting eye. This may be termed amblyopia ex anopsia, and Chavasse 
(1939) distinguished between two different forms of this amblyopia: amblyopia of arrest and amblyopia of extinction. In cases in which the process of inhibition is applied before the establishment of normal full vision, that is in cases of uniocular squint present from birth or developing in early life, the vision which has had no chance of developing is termed "amblyopia of arrest". This differs from "amblyopia of extinction", which represents the vision which has been lost during the process of inhibition but was present at the time of development of the squint. It follows that amblyopia is particularly dense in patients with squints which have occurred early in life, and it is important to note that in a young child suppression is acquired easily because the binocular reflexes are not fully established. It should be realized that the existence of this inhibition during the development of a squint is not a passive capitulation, but a reflex which is brought into play by activity of the visual part of the cerebral cortex. It is unfortunate, however, that such positive action is followed by a negative end-result. In an alternating squint the inhibition is facultative because it persists in the macula of the squinting eye only during the period of active use of the macula of the nonsquinting eye, and changes readily from one eye to the other so that each is capable of functioning normally in turn. This paper is not concerned with the problem of facultative inhibition.

In the second place, in the development of a squint, there may be an alteration in the spatial sense of the retinal receptors of the squinting eye over the whole region of the retina, including the fovea, so that a non-foveal retinal receptor assumes a foveal type of fixation. This type of fixation is termed eccentric fixation, and it is a phenomenon which is confined to a uniocular type of squint. The alternating type of squint, despite the presence of a wellmarked alternating form of suppression, is in visual harmony with its environment, because each fovea in turn holds the centre of attention so that all objects are referred to this central point of reference and there is no stimulus to develop an eccentric type of fixation. There is evidence that an eccentric type of fixation develops more frequently and more rapidly when the uniocular squint occurs at an early age (Worth, 1903). Eccentric fixation, like suppression, is an adaptation of the visual cortex to the presence of an ocular deviation, but it must be realized that it is an unrewarding development because, despite the ability of a non-foveal retinal receptor to change its spatial sense to a foveal form of fixation, there is no change in its visual acuity value so that the eccentric point of fixation retains a low level of vision.

In a discussion of eccentric fixation it is important to consider its relationship to abnormal retinal correspondence. Eccentric fixation is a uniocular phenomenon and implies that fixation of the squinting eye during occlusion of the non-squinting eye is of an eccentric type so that fixation is undertaken by a non-foveal point, although it is likely that eccentric fixation is preceded invariably by an abnormal retinal correspondence. By contrast, abnormal 
retinal correspondence is a binocular phenomenon and implies the establishment of a new series of retinal correspondences in the squinting eye during the use of the two eyes together. It is obvious that the abnormal retinal correspondence of the squinting eye must be centred in some retinal point other than the fovea, but this is not necessarily the point used for fixation during occlusion of the non-squinting eye, so that abnormal retinal correspondence may or may not be associated with an eccentric fixation. The importance of the association and yet independence of eccentric fixation and abnormal retinal correspondence establishes the fact that the restoration of true fixation by the elimination of eccentric fixation does not necessarily alter an accompanying abnormal retinal correspondence.

\section{Emergence of Pleoptic Methods}

The progress of medicine is often gradual, but sometimes rapid advance is made due to the development of a new idea. Such an advance in the understanding and treatment of amblyopia is seen in the study of pleoptics which presents a new approach to the problem. The word pleoptics, which means "full vision", is used to describe certain methods of treatment of amblyopia ex anopsia and of anomalies of fixation, which aim at restoring normal visual acuity and normal fixation in the affected eye. The term was applied originally by Bangerter (1953), who described such a method of treatment. By this method the diagnosis of eccentric fixation is made by asking the patient to fix a point of light which is projected on to the fundus by an ophthalmoscope, and any fixation which is not strictly foveal is regarded as eccentric. The treatment of eccentric fixation aims at a functional destruction of the eccentric point of the deviating eye by dazzling or blinding it with a light of high intensity, followed by intermittent stimulation of the true fixation area (that is, the fovea) by a flashing light which enters the eye through a modified Gullstrand ophthalmoscope. This passive method of treatment is followed by active methods in which the patient is taught to recognize a true type of foveal fixation. Many instruments are used in such treatment, and emphasis is laid on the importance of utilizing the play instincts of the child and of involving the use of the hands with the visual functions.

More recently, a different method of treatment of amblyopia and of anomalies of fixation has been introduced by Cüppers (1956), and his method forms the basis of this paper. Cüppers emphasizes the importance of establishing a clear understanding of the exact nature of a case of eccentric fixation before proceeding with its treatment, and for this reason the methods of diagnosis and treatment are discussed separately. There are, however, two aspects of the subject-the role of occlusion and the assessment of the visual acuity-which are concerned with both diagnosis and treatment, and these will be detailed in the first place. 


\section{Role of OcClusion in EcCentric Fixation}

It is now widely recognized that occlusion of the non-squinting eye is of no value in restoring vision in the squinting eye once that eye has acquired an established form of eccentric fixation. It is interesting to recall that Chavasse (1939) showed that occlusion is rarely successful in patients in whom a squint had developed in the first year of life, but that the percentage of success increased with the age at onset, so that occlusion was successful in 93 per cent. of squints which occurred after the age of 5 years; it is almost certain that this relative failure of occlusion in squints of early onset is due to the presence of an eccentric type of fixation. It is suggested, however, that occlusion of the non-squinting eye may be successful even when the fixation of the squinting eye is regarded as eccentric (Yoxall, 1939; Chinaglia and Amidei, 1955), but it is difficult to accept this finding without the reservation that it is not possible to ascertain a re-establishment of true central fixation without examination by the newer diagnostic instruments, such as the Visuscope.

If such cases exist, it is likely that the occlusion of the non-squinting eye has been adopted at an early stage in the development of the eccentric fixation when the retinal receptors were still in a state of flux, and it would appear that, in any case of established eccentric fixation, occlusion of the non-squinting eye is contraindicated because it merely fosters the eccentric retinal point of the squinting eye. It follows, therefore, that the logical treatment of such cases is to carry out total occlusion of the squinting eye in order to eliminate the persistent use of the eccentric retinal point, and there is evidence that this procedure facilitates the application of pleoptic treatment at some later date. It is of great interest to note, however, that in a few cases of eccentric fixation in children too young to respond to pleoptic methods, a spontaneous but gradual shift of fixation from an eccentric point to a centric one has occurred during prolonged total occlusion of the affected eye. It is possible that this rare event occurs only in cases in which the eccentric fixation is not fully established at the time of occlusion.

Occlusion plays an important part in the diagnostic and therapeutic phases of pleoptic methods. This consists of occlusion of the unaffected eye during the examination and treatment of the affected eye, so that the patient is able to concentrate the attention of the eccentrically fixing eye wholly on the tests. This occlusion also serves the purpose of avoiding any influence which the presence of an abnormal retinal correspondence may have on the fixation of the affected eye. At all other times, however, it is essential to maintain constant occlusion of the affected eye until the successful completion of the treatment. This occlusion is of no value by itself in eliminating eccentric fixation once this is established, but it does prevent the continued use of the eccentric point, and therefore facilitates the disappearance of eccentric fixation following treatment. The re-establishment of central fixation in the affected eye, as a result of treatment, may be followed by occlusion of the 
unaffected eye in order to concentrate attention on the re-established fovea of the affected eye, but it must be emphasized that it is essential to avoid transferring occlusion to the unaffected eye until there is a complete reestablishment of a correct localization of the affected eye, otherwise there may be a tendency for a reversion to the original state of eccentric fixation.

\section{VisuAl ACUITY IN ECCENTRIC FiXation}

In untreated eccentric fixation, unless the eccentric point is adjacent to the fovea, there is a complete loss of central vision due to foveal inhibition. The vision of the eccentric point in such cases is variable; it may be as low as a mere awareness of hand movements or it may be as high as 6/60. In a few cases, however, the eccentric point is close to the fovea and vision may be as high as 6/12 despite a slightly eccentric fixation. The appreciation of letters at a reading range is similarly reduced. During the treatment of eccentric fixation it is important to record the visual acuity at the various stages of treatment, because this is a true measure of foveal function, and the restoration of foveal fixation in no way implies an automatic restoration of foveal function. In cases in which there is an improvement in vision, it is interesting that there is a definite delay in the restoration of the ability to read correctly groups of letters despite the earlier return of the ability to read correctly individual letters of the same size as the letters in the group. It is likely that this peculiarity of vision is related to the phenomenon of visual agnosia and that it represents an upset of a perceptual nature as part of the mechanism of suppression. The visual acuity of individual letters may be termed the "angular visual acuity" to distinguish it from the "cortical visual acuity" which is concerned with a series of letters. Both forms of visual acuity should be recorded at each examination.

\section{Diagnosis of ECCENTRIC Fixation}

The establishment of the diagnosis of eccentric fixation is achieved by the use of several instruments, including the Visuscope, the Euthyscope, and the Coordinator. Information is also obtained by an assessment of the suppression scotoma on the Bjerrum screen. It is possible to diagnose certain cases of eccentric fixation simply by the appearance of the corneal reflections during the application of the cover test, but this is an unreliable method, particularly if the eccentric point of fixation is adjacent to the macula, and confusion may be caused also by the presence of an abnormally large angle alpha.

(1) The Visuscope (Cüppers).-This is a conventional ophthalmoscope incorporating a small star in the path of light within the ophthalmoscope so that the star is directed on to the retina during examination of the fundus, and appears to the observer as a black star. The patient fixes the star and undesirable movements of the eye are avoided by using a relatively low illumination. The purpose of the examination is for the observer to determine the 
area of the retina which is used for fixation by an assessment of the position of the star in relation to the fovea. The fovea is identified readily by a trained observer, but its recognition may be made easier by the use of a green filter. It is possible to carry out the examination with the undilated pupil, but the examination is facilitated by the use of a mydriatic, particularly in young children. If the fixation of the eye under examination is normal, the star is found on the fovea, but if there is any abnormality of fixation the star assumes an extra-foveal position. There are two forms of extra-foveal fixation. First, the star may be fixed by a constant extra-foveal point (Fig. 1a), which may be close to the fovea or some distance from it in any direction, even on the nasal side of the disc. In certain cases it may be difficult to establish whether a point is eccentric or not when it is situated close to the fovea, particularly when fixation is unsteady with quick lateral movements on and off the macula. It is important, therefore, for the examiner to acquire a wide experience of the normal range of variations. Secondly, the star may be fixed by a number of different extra-foveal points over a wide area of the retina so that there is no one constant point of fixation (Fig. 1b). It should be realized, however, that the eccentric point of fixation as seen by the Visuscope may not necessarily correspond with the eccentric point of fixation which the patient uses for straight-ahead projection in space. For example, in a convergent squint with eccentric fixation, the retinal point associated with a straight-ahead projection lies on the nasal side of the fovea, but the retinal point adopted for the fixation of the Visuscope star may approach nearer to the nasal side of the fovea or may even pass to the temporal side of the fovea. In certain of these cases it seems that this point of fixation of the Visuscope star corresponds in position to the border of the suppression scotoma which results from the presence of macular inhibition and which may be plotted on the Bjerrum screen (Fig. 1c), but in other cases the star is not related to the border of the scotoma. This change of retinal fixation

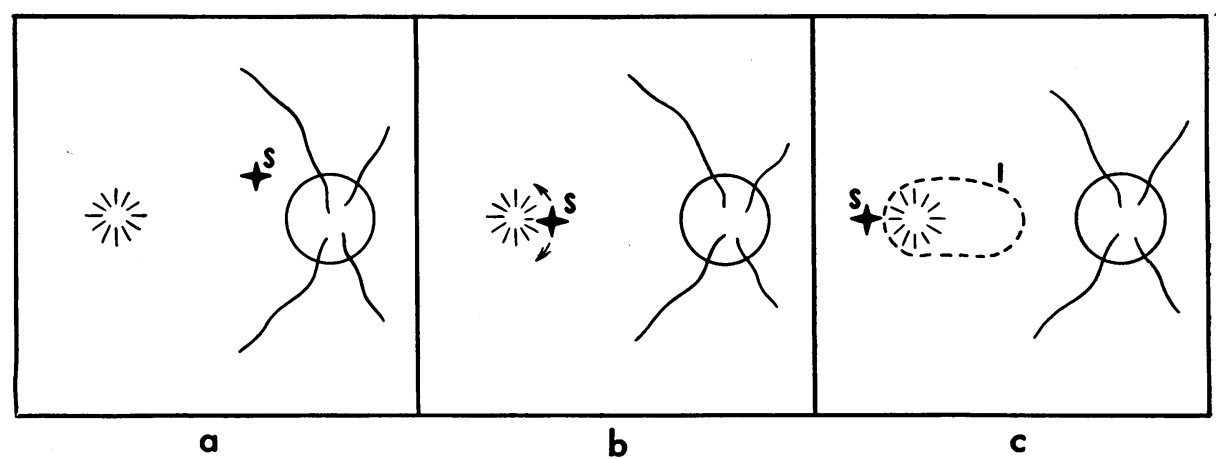

Fig. 1.-Right convergent squint with eccentric fixation. Diagnosis with Visuscope.

(a) Fixation of star (S) by definite retinal point.

(b) Fixation of star (S) over varying retinal area.

(c) Fixation of star (S) at temporal border of area of retinal inhibition (I). 
with the Visuscope may represent an attempt by the retina to obtain a clearer view of the star.

The Visuscope, therefore, provides a method of arriving at a diagnosis of eccentric fixation, and it should be emphasized that it is applicable even to very young children, although special steps should be taken to ensure that the child is concentrating his attention. Repeated instructions to "look at the star" must be given, otherwise fixation may be ill-sustained through lack of attention. It may be logical to conclude that occlusion of the unaffected eye should not be undertaken in any case of uniocular squint without an assessment of the state of fixation of the squinting eye with the Visuscope, although this may be a counsel of perfection.

In some cases of eccentric fixation, the Visuscope is also of value in assessing the state of foveal function. This is concerned with the subjective impression obtained from the patient by directing the star from the Visuscope on to the fovea. If the foveal inhibition is dense the patient fails to see the star, but if there is some foveal function the star is appreciated, although not in the central part of the beam of light because of the abnormal projection of the fovea in association with the adoption of a straight-ahead projection of the eccentric retinal point. It is interesting to note, however, that in certain cases the star appears double through the persistence of some degree of normal foveal projection despite the development of an abnormal foveal projection. The "incorrect" star is thus seen more clearly than the "correct" one.

A further application of the Visuscope is its use in determining the state of binocular retinal correspondence of the foveae of the two eyes. The test is carried out at a distance of $1 \mathrm{~m}$. and, whilst the non-squinting eye is fixing the spot-light at the centre of the Maddox cross (tangent scales) through an, inclined mirror, the star from the Visuscope is directed on to the fovea of the squinting eye. The star is then projected by the patient on to the Maddox cross; a normal state of foveal correspondence is present if the star is centred on the spot-light, but if the star is projected away from the light the angle of anomalous binocular localization is indicated according to the position of the star on the cross. It is obvious that this test is applicable only to cases in which the star from the Visuscope is able to be appreciated by the fovea of the squinting eye, and this implies an absence of complete foveal inhibition. Furthermore, it is a necessary part of the test that the vision of the squinting eye is sufficient to permit steady fixation of the light on the Maddox cross.

(2) The Euthyscope (Cüppers).-This instrument is concerned with the production of after-images from the fovea and from a small area of the surrounding retina of the affected eye. The examination with the Euthyscope is preceded by instillation of a mydriatic in order to achieve full dilatation of the pupil. The Euthyscope is used to project a bright beam of light on to 
an area of the retina which is $30^{\circ}$ in size with the fovea as the central point. At the centre of the illuminated area there is a circular area, $3^{\circ}$ to $5^{\circ}$ in size, which is not illuminated because of the insertion of an opaque disc into the ophthalmoscope. It is essential that this instrument is kept still during use and that there is no movement of the patient's eye so that the centre of the unlit area is maintained on the fovea. The light with its dark centre is projected into the eye for 20 to 30 seconds. The patient then directs his eye to a white screen with a central cross, and he is instructed to observe the appearance of the after-image and to describe its relation to the central cross.

The appearance of an after-image is a well-known physiological phenomenon, and demonstrates the fact that stimulation of the retina produces a response which is not limited in time to the duration of the stimulus. The primary image, which is the immediate result of the stimulus, is followed by an after-image which may be of two types: first, a positive after-image, which is a persistence of a physiological process of the same type as that which produces the primary image, so that it appears as a bright $30^{\circ}$ area with a dark $3^{\circ}-5^{\circ}$ central area, and secondly, a negative after-image, which is a persistence of a physiological process of the opposite type to that which produces the primary image, so that it appears as a dark $30^{\circ}$ area with a bright $3^{\circ}-5^{\circ}$ central area. In the normal subject the negative phase may follow the positive phase three or four times after one stimulation, and the whole process may persist for as long as 30 minutes. The observation of an afterimage may be difficult, particularly in an eye with marked macular suppression, but its appearance may be enhanced by the use of an Alternoscope, which provides regulated, but variable, light and dark phases on the viewing screen by means of a flashing light. This flickering light results in blinking, and the rapid movements of the lids cause an accentuation of the after-image, particularly the negative one. It is usually found that the denser the suppression the shorter must be the light phase of the Alternoscope.

In eccentric fixation, the central spot of the after-image, whether seen in the positive or negative phase, is projected away from the central cross on the screen because of the straight-ahead localization of the eccentric retinal point (Fig. 2, opposite).

In a convergent squint, the projection is to the side opposite to the squinting eye, and in a divergent squint the projection is to the same side as the squinting eye, that is, the direction of the visual projection is the same as the direction of the squint. For example, in a right convergent squint the projection is to the left (Fig. 2), and in a right divergent squint the projection is to the right. The Euthyscope, therefore, provides useful diagnostic information in cases of eccentric fixation, but it is also of value in determining 
the chances of successful treatment, because there is evidence that the ready ability of the patient to convert a positive after-image to a negative one is a favourable sign.

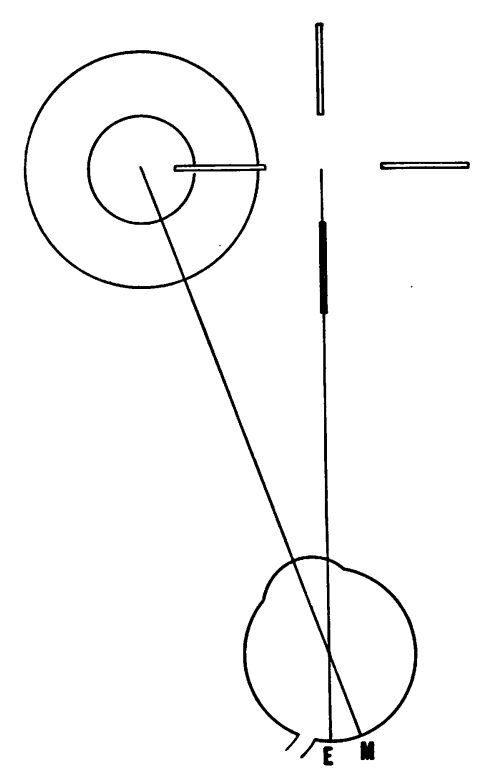

Fig. 2.-Right convergent squint with eccentric fixation.

Diagnosis with Euthyscope and screen with fixation cross .

Straight-ahead localization of eccentric retinal point $(\mathrm{E})$. Projection of after-image from macula (M) to left.

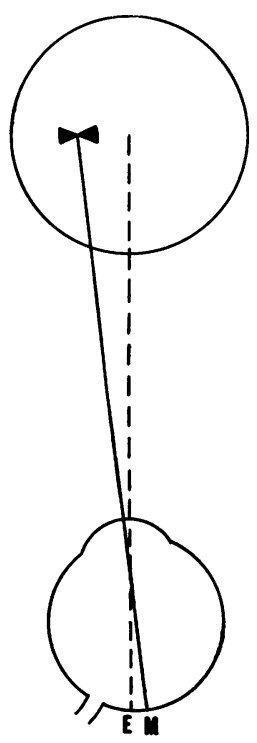

Fig. 3.-Right convergent squint with eccentric fixation.

Diagnosis with Coordinator.

Straight-ahead localization of eccentric retinal point $(E)$. Projection of brush from macula (M) to left.

(3) The Coordinator (Cüppers).-This instrument makes use of the entoptic appearance of Haidinger's brushes during the passage of polarized light rays into the eye through a Nicol prism. This physiological phenomenon is probably the result of the double refracting action of the parallel arrangement of the radial fibres of Henle's layer in the parafoveal part of the retina. It follows that the brushes are visible only in cases in which there is some degree of macular function, so that a failure to appreciate the brushes signifies a dense inhibition of the macula. The presence of some degree of macular function usually indicates that the point of eccentric fixation lies close to the fovea, and, conversely, the presence of dense inhibition of the macula usually indicates that the point of eccentric fixation lies at some distance from the fovea. In cases of eccentric fixation showing an appreciation of the brushes, they are projected away from the central target because the eye is regarding the target with the point of eccentric fixation (Fig. 3). The ability of the patient to change the localization of the brushes so that they are projected on to the target is a favourable sign for successful treatment. 
(4) Determination of the Suppression Scotoma.-The development in the squinting eye of an eccentric type of fixation implies the presence of a state of inhibition of the macular area. The extent of this inhibition may be determined by plotting the scotoma on the Bjerrum screen during the fixation of the central target of the screen with the eccentric retinal point. It is obvious, of course, that this method of examination is possible only when the point of eccentric fixation is able to maintain fixation.

\section{Treatment of EcCentric Fixation}

This involves the use of several instruments which vary according to whether the treatment is uniocular or binocular in its application. The uniocular forms of treatment are concerned with the Euthyscope, the Coordinator, and with various forms of apparatus which provide visual exercises. The binocular forms of treatment are concerned with the incorporation of after-images and of Haidinger's brushes in the synoptophore, and finally with the conventional methods of treating squint with the ordinary synoptophore.

(1) The Euthyscope (Cüppers).-This instrument is used in the treatment of eccentric fixation and its particular importance lies in its use in cases which show an inhibition of foveal function because such patients are not suitable for treatment with the Coordinator. The treatment with the Euthyscope is based on the concept that an eye with eccentric fixation is unable to fix with the fovea an object in space, either spontaneously or even after occlusion of the sound eye. It is essential, therefore, to create a process in the macula which results in the formation of an image in space, and then to produce a re-orientation of the cerebral cortical processes so that the image is appreciated in the correct line of projection. As discussed earlier in this paper, the after-image produced by the Euthyscope is concerned with the fovea and with an area of retina $3^{\circ}-5^{\circ}$ around the fovea, and this implies that the eccentric point must not lie nearer than $3^{\circ}$ to the fovea, otherwise the stimulation of the fovea is associated with a similar stimulation of the eccentric point. In fact most cases which are suitable for treatment with the Euthyscope show an eccentric point which is fairly remote from the fovea, and it is usual to use a disc which causes the production of after-images from an area of retina $5^{\circ}$ around the fovea, but this may be reduced to $3^{\circ}$ if the eccentric point lies between $3^{\circ}-5^{\circ}$ from the fovea.

The first aim in treatment with the Euthyscope is to restore the true spatial value of the macula so that stimulation of the macula results in a straightahead projection. In eccentric fixation the patient projects the after-image away from the central cross on the white screen because of a persistence of the straight-ahead localization of the eccentric retinal point, so that he thinks that his eye is looking straight-ahead despite its convergent position (Fig. 2), but with time and concentration the position of the after-image on the screen 
is changed so that it comes to lie on the cross. It is common experience that a stage is reached in this treatment when the patient is able to centre the afterimage correctly but feels that he is looking to the side of the target; in the case of a right convergent squint the patient feels that he is looking to the right (Fig. 4), and in the case of a right divergent squint the patient feels that he is looking to the left. This is an important stage in the treatment with the Euthyscope because it implies a restoration of a correct localization in space of the fovea despite the persistence of some spatial information from the original eccentric point of fixation so that the eye feels as though it is directed in the visual axis of the eccentric point (Fig. 4). In such cases the patient must be encouraged to appreciate that he has achieved a straight-ahead projection, and it is interesting that children sometimes achieve recognition of this more quickly than adults. Emphasis is laid throughout treatment on the importance of improving the coordination of the hand and the eye, and the patient is told to touch the image with his hand. In the early stages of treatment the distance between the patient and the screen should be about one-third of a metre in order to simplify the test and to enable the patient to touch the screen with his hand, but in the later stages of treatment, once the true spatial value of the macula is restored with a normal type of fixation, the distance is increased in order to enhance the effect. When the after-image has disappeared the Alternoscope is switched off and the patient is instructed to close his eyes tightly for a few seconds. This may revive the after-image and treatment may then be continued. No further application is made with the Euthyscope until the original after-images have completely disappeared.

FIG. 4.-Right convergent squint with eccentric fixation. Treatment with Euthyscope and screen with fixation cross. Straight-ahead projection of after-image from macula (M). Localization of eccentric retinal point (E) to right.

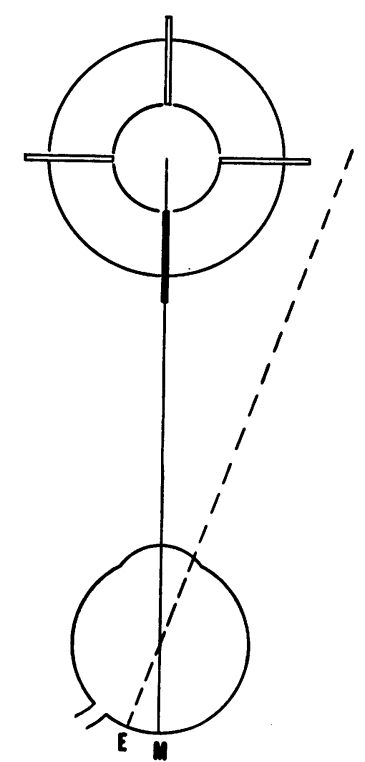

When the true spatial value of the macula has been restored, attention should be directed to the problem of improving the central visual acuity, and it is essential to utilize the negative phase of the after-image for this part of the treatment. In a normal subject the negative after-image appears rapidly, but in a patient with established amblyopia the negative after-image may be achieved with difficulty, and the time taken for the reversal of the after-image varies according to the density of the suppression. It must be 
realized that the light from the Euthyscope increases the excitation of the part of the retina surrounding the macula, but by virtue of the central black spot which protects the macular area there is a decrease in the excitation of the macular area and an enhancement of its amblyopic state. It is necessary to reverse this undesirable state of affairs by an appreciation of the negative after-image, and it is possible to view objects which are placed on the screen through this clear central zone. This is the essence of the treatment with the Euthyscope because it provides a method of overcoming the amblyopia of the macular region, and the prognosis for the treatment of such cases depends to a large extent on the facility with which it is possible to create a negative afterimage. Initially, it is important to present the subject with fairly large single letters and to have a working distance of about one-third of a metre, but, once there is some improvement in the visual acuity, smaller letters may be used and the working distance may be increased. In the later stages of treatment it is possible to use groups of letters.

(2) The Coordinator (Cüppers).-The treatment of eccentric fixation with the Coordinator represents an important phase of treatment because it is concerned directly with the restoration of macular and foveal functions, but its dependence on the appearance of Haidinger's brushes obviously limits its application to cases in which there is normal function of the macula or paramacular region. The Coordinator is of value in cases with marked eccentric fixation in which some degree of macular fixation has been restored by treatment with the Euthyscope, although the fixation may be unsteady. Its main value, however, is in cases in which the point of eccentric fixation lies within $3^{\circ}$ of the fovea before starting treatment, such cases being unsuitable for treatment with the Euthyscope because the central dark area formed by the Euthyscope includes the eccentric point in addition to the fovea. In the different phases of treatment with the Coordinator the use of the diaphragm (Cüppers, 1958) is of particular importance; initially, a small aperture is used in order to limit the area of the macula stimulated by the polarized light, but in the later stages the aperture is increased in size.

Treatment with the Coordinator is dependent on an appreciation of the brushes within the instrument and on an ability to project these brushes on to the centre of a target. It is likely that in the early stages of treatment the brushes will appear to be projected away from the central area because of a persistence of a straight-ahead localization of the eccentric retinal point (Fig. 3), but with time and concentration projection becomes accurate, although for a period there may be an impression, despite the centring of the brushes, that the eye is directed beyond the point of fixation because of the persistence of an impression that the eccentric point has a straight-ahead type of projection (Fig. 5, opposite). In the initial stages of treatment the brushes may disappear or become blurred, but they are maintained more easily in a central position by reducing the size of the diaphragm. The aperture may be 
gradually widened provided the brushes are maintained in a central position but it is interesting to note that the brushes may appear double at this stage. This is because the wide aperture allows the simultaneous appreciation of the true foveal projection and of the persisting false foveal projection (Fig. $6 a$ ). This double foveal projection may be avoided by narrowing the aperture in order to obscure the clear but incorrect image (Fig. 6b) until there is a full restoration of normal foveal projection.

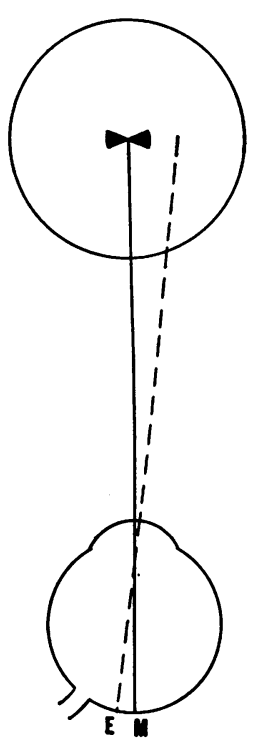

Fig. 5.-Right convergent squint with eccentric fixation.

Treatment with Coordinator.

Straight-ahead projection of brush from macula (M). Localization of eccentric retinal point (E) to right.
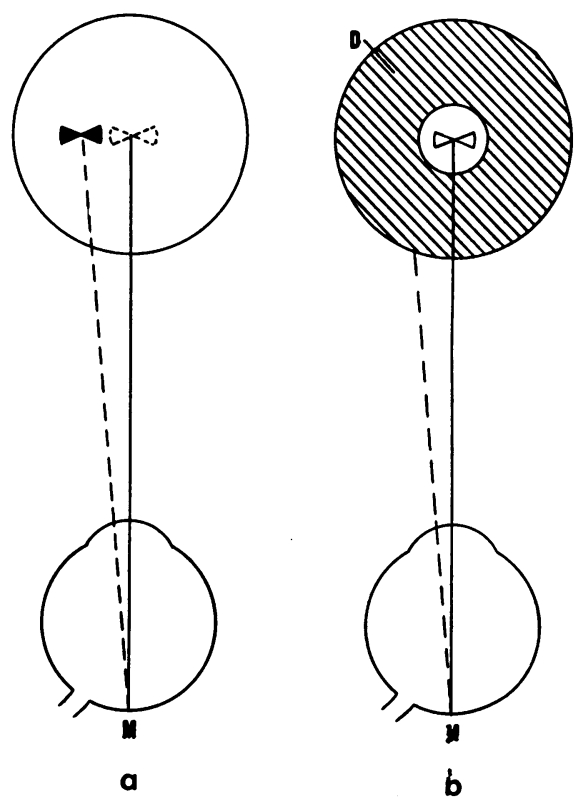

FIG. 6.-Right convergent squint with eccentric fixation. Double foveal projection of Haidinger's brushes (Coordinator).

(a) Straight-ahead projection of brushes from macula (M)-correct brushes but blurred. Projection of brushes from macula (M) to left-incorrect brushes but clear.

(b) Straight-ahead projection of brushes from macula (M)-correct brushes. Incorrect brushes hidden by diaphragm (D).

When true foveal fixation has been established with a normal sense of spatial values, it is important to enhance it by relating the eye to other forms of motor coordination, particularly to movements of the hand. This may be achieved by projecting the brushes on to different parts of the target and at the same time by touching these parts with a pointer controlled under direct view by the hand. In the early stages of treatment it is likely that the pointer will be directed away from the brushes, that is, away from the centre of the target, because of a persistence of fixation of the pointer by the eccentric retinal point (Fig. 7a). The aim of treatment is to superimpose the 
pointer on the brushes, but it should be noted that it is possible at this stage of treatment to find an apparent superimposition of the pointer on the brushes and yet a persistence of the use of the eccentric retinal point for the fixation of the pointer. This is because the eccentric retinal point has retained a foveal type of projection, so that there is an apparent superimposition of the pointer and the brushes (Fig. 7b). This false type of superimposition is due to the identical localization of two different retinal points, and it may be avoided by the use of the small aperture of the diaphragm because this precludes the stimulation of the eccentric point by an object within the visible area (Fig. 7c). Treatment is continued until the patient is able to obtain correct superimposition of the pointer and the brushes despite the use of a large aperture (Fig. 7d). Subsequent treatment with the Coordinator consists in teaching the patient to project the brushes on to various dots or letters which are identified in order to facilitate the re-establishment of central vision.
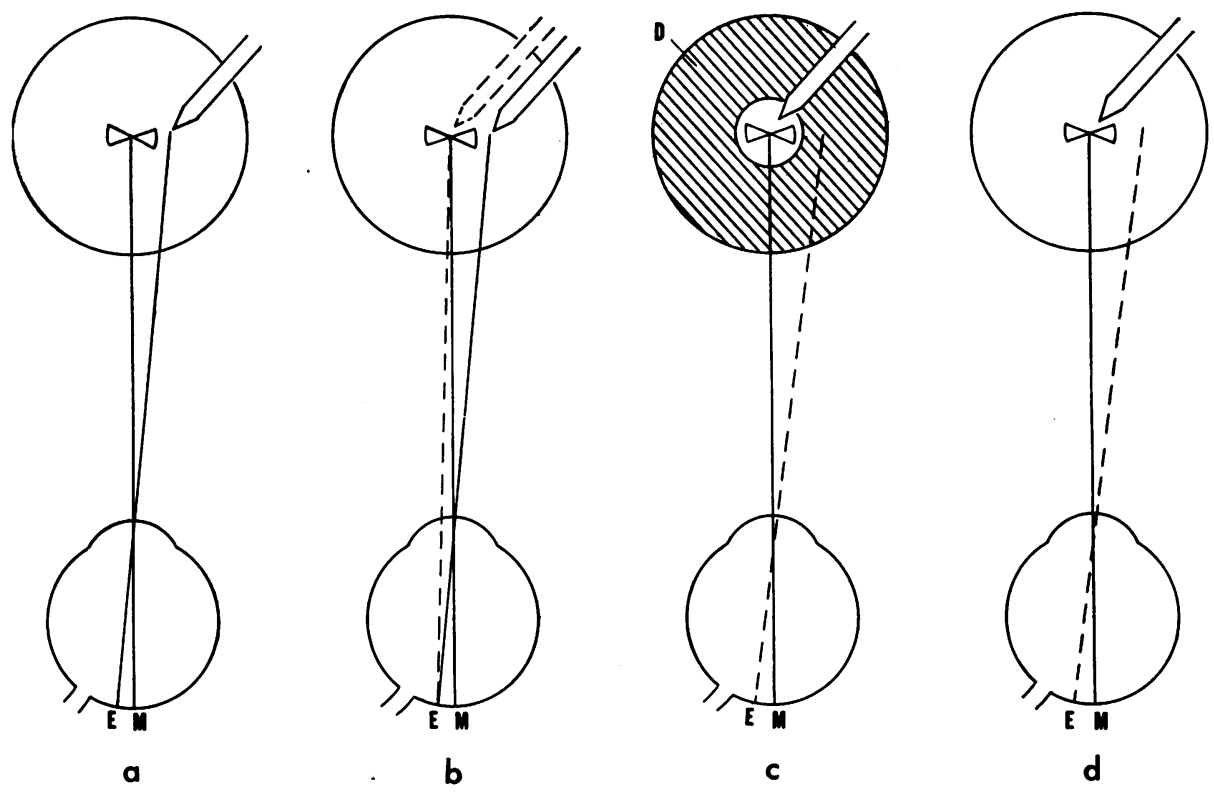

Fig. 7.- Right convergent squint with eccentric fixation.

Identical localization of two different retinal points (Coordinator with pointer).

(a) Separation of brushes and pointer; brushes projected straight ahead by macula (M), pointer fixed by eccentric retinal point (E).

(b) False superimposition of brushes and pointer; brushes projected straight ahead by macula (M), pointer fixed by eccentric retinal point (E) with foveal type of projection (dotted line).

(c) Correct superimposition of brushes and pointer; brushes projected straight ahead by macula (M), pointer fixed by macula (M). Small aperture of diaphragm (D) precludes stimulation of eccentric retinal point $(\mathrm{E})$.

(d) Correct superimposition of brushes and pointer; brushes projected straight ahead by macula (M), pointer fixed by macula (M). Inhibition of eccentric retinal point $(\mathrm{E})$ despite wide aperture of diaphragm. 
A further application of the Haidinger's brushes method is the use of an instrument which permits the brushes to be projected into space instead of being appreciated within the Coordinator. The brushes are projected on to a brightly-lit white magnetic screen on which it is possible to fix letters of different sizes in any position with varying degrees of separation. The first stage of this treatment is to learn to project the brushes on to the centre of the screen and, as in treatment with the Coordinator, the use of the small aperture limits the area of retinal stimulation initially, but later there is a gradual opening of the diaphragm with a maintenance of the correct projection of the brushes. The second stage of treatment is to superimpose the correctly projected brushes on to a single letter on the screen with a simultaneous identification of the letter. The patient is placed at varying distances from the screen. The diaphragm is also used during this treatment. Groups of letters may be used and their positions may be varied according to the area of inhibition and false localization present. In the early stages of treatment two letters may be appreciated; one letter which is seen by the macula and on which the brushes are superimposed, and a second letter which lies to the side of the brushes and is seen by the eccentric retinal point (Fig. 8a). In the later stages of treatment the enhancement of the function of the macula is associated with an inhibition of the eccentric retinal point so that only one

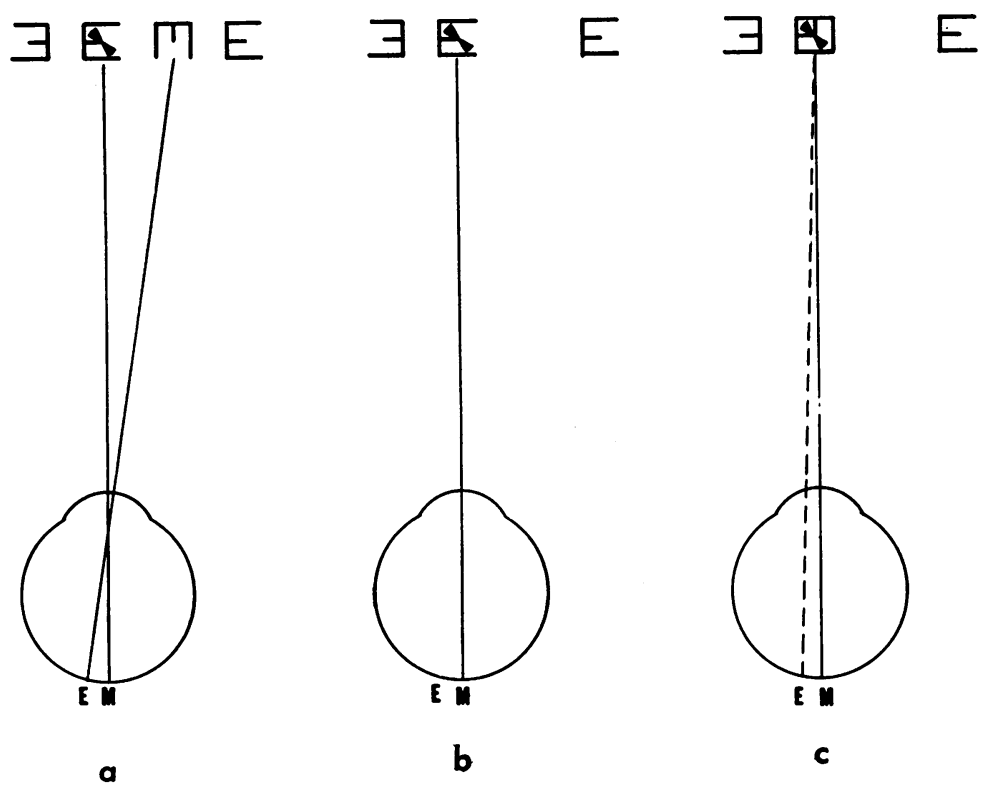

FIG. 8.-Right convergent squint with eccentric fixation.

Line of letters viewed through Haidinger's brushes in space.

(a) Subjective appreciation of two letters; one by macula (M), one by eccentric retinal point (E).

(b) Subjective appreciation of one letter only by macula (M). Inhibition of eccentric retinal point (E).

(c) Subjective appreciation of two superimposed letters; one by macula (M), one by eccentric retinal point (E) with foveal type of projection (dotted line). 
letter is visible, namely that seen by the macula, with a failure to appreciate the letter stimulating the eccentric retinal point (Fig. $8 b$ ). It should be noted, however, that failure to see the second letter which lies to the side of the brushes may be associated with the superimposition of two letters on the brushes, and this is due to the persistence of a foveal type of projection of the eccentric retinal point (Fig. 8c). This false superimposition of the two letters is due to the identical localization of two different retinal points, and may be considered as a form of uniocular diplopia despite the fact that the two objects appreciated simultaneously are not separated from one another. A further form of uniocular diplopia may become apparent during this treatment through a persistence of a false type of foveal projection despite the re-establishment of a true type of foveal projection. This is illustrated by the observations of a patient with a right convergent squint and eccentric fixation who noticed, on projecting the brushes on to the letter " $E$ " at a distance of $3 \mathrm{~m}$. (Fig. 9a), that the clear " $\mathrm{E}$ " moved to the right and that a blurred "E" moved in behind the brushes from the left (Fig. 9b). It may be assumed that the blurred " $E$ " represents a persistence of the false foveal type of projection of the eccentric point, whereas the clear " $E$ " represents the true projection of the same point. On attempting to move the correct projection of the " $E$ " on the right back to the centre, both " $E$ "s moved simultaneously to the left, the brushes remaining on the blurred "E" (Fig. 9c). On a second attempt to place the brushes on the clear projection of the "E" at the right, both the brushes and the blurred "E" moved to the right (Fig. 9d) and the two "E"s then joined (Fig. 9e) and jumped back into the centre with the brushes (Fig. $9 f$ ).

Experience in the treatment of cases of eccentric fixation with the Coordinator has shown that there may be no absolute correlation between the re-establishment of central fixation and the return of full foveal vision. It is essential to concentrate attention on restoring a normal type of fixation before making any attempt to improve the vision to a normal level, but there is evidence that, even after the full restoration of foveal fixation, there may be a comparative failure in the improvement of central vision.

(3) Visual Exercises.-In cases in which there is a restoration of some degree of central vision following the re-establishment of central fixation, it is important to improve the vision further by the application of various visual exercises, many of which may be carried out at home. The exercises are directed largely to the appreciation of the separation of closely related objects and involve the mechanism of cerebral coordination because of the use of the hand with the visual processes. Examples of such exercises are the crossing out of a particular letter throughout a page of print, or the pricking of a picture formed by dots. The separation between the letters and dots may be decreased as the visual acuity improves until a normal level of vision is attained. 

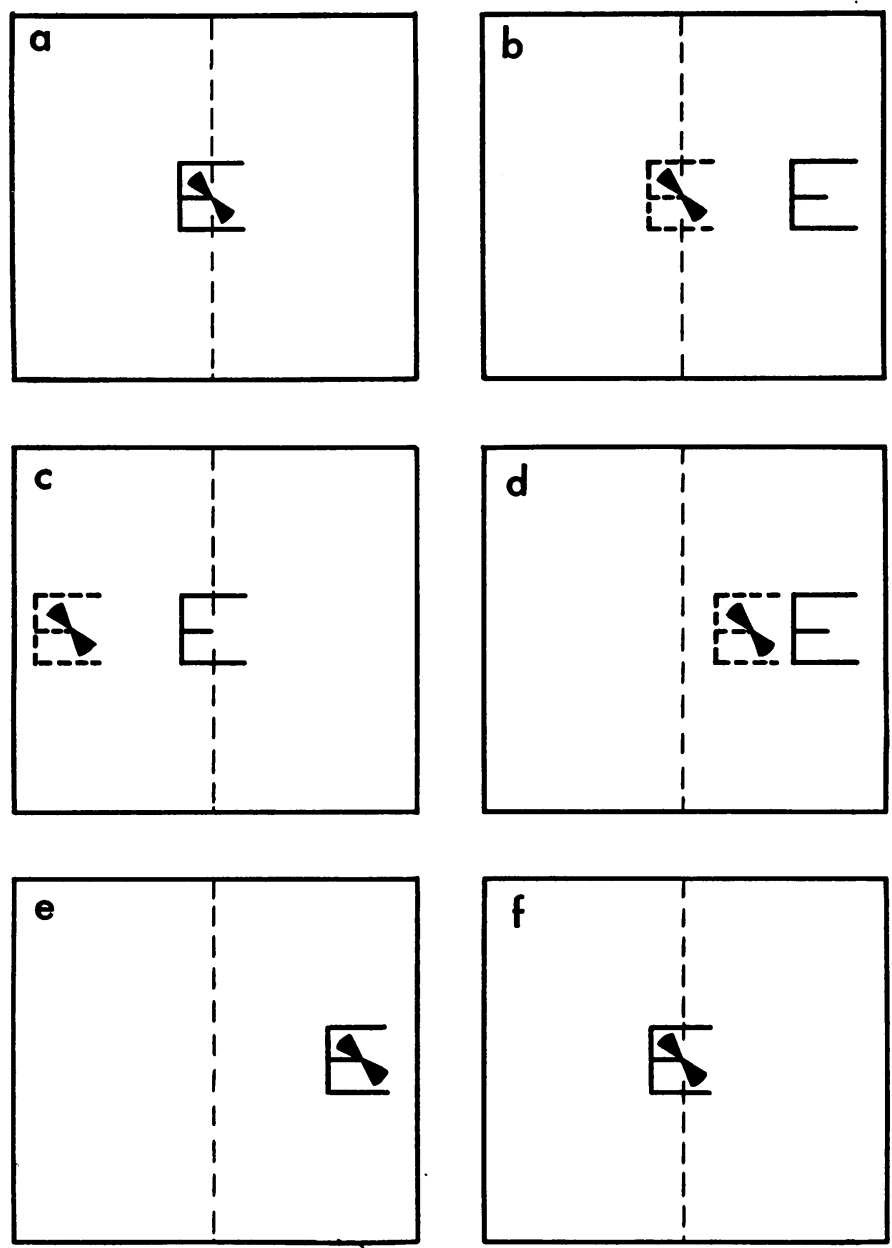

FIG. 9.-Right convergent squint with eccentric fixation. Uniocular diplopia with Haidinger's brushes in space.

(4) Restoration of Binocular Function.-It is natural that the emphasis of treatment for eccentric fixation is on uniocular methods because the main purpose of such treatment is the restoration of a normal type of fixation and of a normal type of vision in the squinting eye. Such a method is of great value when it is applied to a squinting eye with eccentric fixation following the loss of function in a normal non-squinting eye through trauma or disease, but this represents only a small number of cases. The essential aim of treatment in the majority of cases of squint with eccentric fixation is the development of binocular function, and this must follow closely on the re-establishment of correct uniocular fixation. The method of binocular training 
involves the incorporation of after-images, of a vertical and horizontal type, in the synoptophore in order to stimulate a correct binocular localization from both maculae. This treatment results in a gradual decrease in the angle of abnormal binocular localization, but it is essential to continue the treatment with a method involving the incorporation of Haidinger's brushes in the synoptophore, because such a method is concerned directly with the restoration of true foveal values. The ability to superimpose correctly the after-images and the brushes is followed by the use of real images, but there may be some initial difficulty in this phase of the treatment because of a reversion to an abnormal type of binocular localization. The use of the diaphragm in the early stages of the treatment with real images represents a way of overcoming this difficulty by preventing stimulation of the area of abnormal localization.

\section{Discussion}

There is no doubt that pleoptic methods play an important role in the treatment of amblyopia associated with an eccentric type of fixation, but there are certain factors which, in the present state of knowledge, limit their satisfactory application.

In the first place, pleoptic treatment is seldom possible in children under the age of 6 years because by its very nature it demands a high degree of sustained concentration and cooperation. It is relatively easy for a young child to take part in the many forms of pleoptic games which are designed for uniocular training, but this treatment is of no value until central fixation is restored and the success of such treatment in young children with amblyopia is limited to those with a normal type of fixation. This is an important point from a statistical point of view, and may account for the discrepancy in the incidence of so-called eccentric fixation in different clinics, and for discrepancies in the results of pleoptic treatment. It would appear to be correct to include in a discussion of eccentric fixation only cases in which a nonfoveal retinal area is used for fixation, as demonstrated by the diagnostic use of the Visuscope, Euthyscope, and Coordinator, and to exclude cases showing loss of central vision without the establishment of an eccentric fixation. The only rational line of treatment in eccentric fixation in young children is total occlusion of the squinting eye in order to remove the persistent practice of eccentric fixation, and to follow this by the application of treatment for the eccentric fixation as early as possible. This form of occlusion undoubtedly facilitates the conduct of such treatment and has the additional merit in a few cases of producing a spontaneous restoration of central fixation during the period of occlusion, as discussed in an earlier part of this paper.

In the second place, there is evidence that, although pleoptic treatment may be successful in restoring a central type of fixation in the squinting eye, it may not be successful in some cases in restoring a good degree of central vision. It would appear, therefore, that the original view of Chavasse (1939), 
that there may be a recovery of the amblyopia of extinction but not of the amblyopia of arrest, remains unchanged, despite the advent of newer methods of treating amblyopia. It is possible, however, that the restoration of a central type of fixation, even in the absence of any significant improvement in central vision, may be a great advantage in the surgical treatment of the squint. It is well known that it is difficult to obtain a satisfactory surgical result in cases with eccentric fixation, but it would appear that a good cosmetic result is facilitated by the restoration of central fixation despite the persistence of amblyopia.

The introduction of pleoptic methods had led to a renewed interest in the problem of the treatment of eccentric fixation in squint. It is important, however, that this interest in treatment should not divert attention from the necessity of trying to prevent the development of the condition. There is little doubt that in uniocular squints occlusion of the non-squinting eye, within as short a time as possible of the onset of the squint, is the most effective way of reducing the degree of amblyopia and of preventing the development of the associated phenomenon of eccentric fixation. Certainly there is no place for a complacent attitude in the management of cases of uniocular squint, particularly in very young children.

This paper is based on a joint communication to the Section of Ophthalmology of the Royal Society of Medicine, London, on November 13, 1959. We should like to acknowledge the help of Mr. T. Keith Lyle and our other colleagues in the Orthoptic Department of Moorfields Eye Hospital (High Holborn Branch) during the preparation of this paper. Our thanks are due also to Miss Jane Walker who prepared the original diagrams for the meeting of the Royal Society of Medicine, and to Mr. T. Tarrant of the Medical Illustration Department of the Institute of Ophthalmology, London, who prepared the diagrams for this paper.

\section{REFERENCES}

Bangerter, A. (1953). Ophthalmologica (Basel), 125, Suppl. 37.

Chavasse, B. (1939). "Worth's Squint", 7th ed. Ballière, Tindall, and Cox, London.

Chinaglia, V., and Amidei, B. (1955). Ann. Ottal., 81, 563.

CüPPERS, C. (1956). Klin. Mbl. Augenheilk., 129, 579. (1958). Private communication.

Worth, C. (1903). " "Squint. Its Causes, Pathology, and Treatment." Ballière, Tindall, and Cox, London.

Yoxall, I. (1939). Brit. orthopt. J., 1, 15. 